\title{
Rursus
}

Russus

Poiétique, réception et réécriture des textes antiques

$4 \mid 2009$

Erotica

\section{Défense et illustration d'Amour en poésie dans les Tristes d'Ovide et dans les Schediasmata Poetica du poète néo-latin Paul Schede Melissus}

\section{Karine Descoings}

\section{OpenEdition}

Journals

Édition électronique

URL : http://journals.openedition.org/rursus/265

DOI : 10.4000/rursus.265

ISSN : 1951-669X

Éditeur

Université Nice-Sophia Antipolis

Référence électronique

Karine Descoings, «Défense et illustration d'Amour en poésie dans les Tristes d'Ovide et dans les Schediasmata Poetica du poète néo-latin Paul Schede Melissus », Rursus [En ligne], 4 | 2009, mis en ligne le 01 février 2009, consulté le 19 avril 2019. URL : http://journals.openedition.org/rursus/265 ; DOI : 10.4000/rursus.265

Ce document a été généré automatiquement le 19 avril 2019.

Rursus 


\title{
Défense et illustration d'Amour en poésie dans les Tristes d'Ovide et dans les Schediasmata Poetica du poète néo-latin Paul Schede Melissus
}

\author{
Karine Descoings
}

\section{Introduction}

1 Exilé en 8 après J.-C. par Auguste, qui lui reprochait l'immoralité de l'Art d'Aimer, Ovide s'employa, dès son premier recueil d'exil, les Tristes, à rédiger un long plaidoyer pour essayer de se défendre ou, du moins, de réduire les accusations portées contre lui. Les 578 vers du deuxième livre se concentrent notamment, à partir du vers 239 , sur l'examen et la réfutation des griefs formulés à l'encontre du poète, accusé « d'avoir, en composant un poème infâme, professé l'abject adultère ${ }^{1}{ }^{\prime}$. En rédigeant sa défense en vers, NasonOvide entendait convaincre ses lecteurs qu'en devenant le maître de l'Amour en littérature, il n'avait commis nul crimen, rien qui ne souffre l'accusation.

2 Des siècles plus tard, dans l'Allemagne récemment convertie au protestantisme, deux poètes néo-latins allemands, Petrus Lotichius Secundus (1528-1560) et Paul Schede Melissus (1539-1602) furent contraints de louvoyer entre deux écueils ou, plutôt, de faire coexister dans leurs œuvres deux aspirations difficilement conciliables: quoiqu'ils voulussent suivre les traces des élégiaques romains, fidèles serviteurs d'Amour, ils ne pouvaient faire fi des impératifs moraux que la religion réformée faisait peser sur la littérature contemporaine. Les critiques et les reproches qu'essuyèrent leurs entreprises littéraires conduisirent Melissus, quelques années après la mort de Lotichius, à adresser une longue pièce en distiques élégiaques au meilleur ami du défunt, Johannès Hagen. L'élégie, publiée d'abord en 1583 à Nuremberg, puis incluse dans la deuxième édition des 
Schediasmata Poetica parue en 1586 à Paris, a pour titre Ad Iohannem Hagium. Amorem diuinitus esse ingeneratum in hominibus, unde Poetarum scriptis decantatissimus sit, et pour sous-titre, dans la première version de la publication, Defensio et Lotichii et auctoris ${ }^{2}$. Ce sous-titre n'est pas sans rappeler le projet de la pièce ovidienne des Tristes : le texte de Melissus se distingue d'ailleurs de la recusatio traditionnelle que l'on peut lire sous la plume d'un Ronsard, modèle du poète allemand, dans l'« Élégie à Cassandre » des Amours. Pour expliquer pourquoi il a choisi de s'en tenir à la veine érotique plutôt que d'entreprendre le travail de longue haleine qu'exigerait un éloge des hauts faits du roi de France, Ronsard ne prétexte que la difficulté de l'entreprise, qui excéderait son talent. Ovide et Melissus, en revanche, doivent affronter les exigences d'un pouvoir qui ne se borne plus à leur demander de donner à leurs vers un contenu politique : il entend aussi les contraindre à exercer une censure morale sur leurs œuvres.

3 Nous nous proposons donc d'étudier ces textes en parallèle, pour tenter de voir quelle stratégie argumentative est déployée par chacun de ces auteurs afin de se défendre d'avoir consacré leurs poèmes à l'Amour. Nous mettrons en évidence les points de convergence entre ces plaidoyers sans passer sous silence la singularité de Melissus et les réinterprétations qu'il fait subir à la poésie antique pour adapter son argumentation aux circonstances et aux particularités de son époque. Cette étude devrait nous permettre de dégager les spécificités d'Eros dans l'humanisme protestant.

\section{Ovide ou la revendication de la liberté d'Amour en poésie}

4 Le plaidoyer ovidien est sous-tendu par une argumentation juridique : Nason affirme que son œuvre ne s'oppose en rien aux commandements des lois (non tamen idcirco legum contraria iussis ${ }^{3}$ ) et rappelle les précautions dont il s'était entouré dans l'Art d'aimer, en excluant de son lectorat les femmes mariées de naissance libre :

Nil nisi legitimum concessaque furta canemus,

Inque meo nullum carmine crimen erit ${ }^{4}$.

5 L'emploi de l'adjectif legitimum, du participe concessa et du nom crimen paraît pourtant souligner l'emprise d'un pouvoir coercitif sur un domaine qui devrait ressortir de la vie privée, l'Amour. Au demeurant, malgré cet arsenal répressif, l'oxymore concessaque furta montre que la matière érotique demeure intrinsèquement subversive, bien qu'on tente de l'encadrer et de lui fixer des limites légales. Pour chanter l'Amour tel que le conçoit l'élégie érotique, le poète est alors contraint de louvoyer entre des interdictions, évoquées par la multiplicité des négations, et de chercher les brèches, les ultimes interstices que laisse un prince désireux de régenter jusqu'à la littérature. En effet, les guerres civiles ayant saigné Rome à blanc, l'empereur avait entrepris, en promulguant les lois juliennes, de mener une politique nataliste, en encourageant le mariage, jusqu'à la contrainte si nécessaire. Il entendait que la littérature le soutienne dans cette entreprise et chante les avantages du mariage avec une épouse honorable, et non les plaisirs trouvés à entretenir des relations illégitimes avec des femmes aux mœurs légères. Si ce genre de relations a toujours été toléré à Rome, la marge de liberté tendait à se réduire, aussi l'écrivain devait-il se tenir prêt à se défendre et à argumenter pour soutenir son droit à chanter en poésie des relations qui menaçaient celles que l'empereur voulait voir promues. 
6 L'autocitation vient souligner le durcissement de la législation morale augustéenne : en vérifiant dans l'Art d'aimer, le lecteur peut constater que le poète n'annonçait nullement qu'il chanterait « ce que la loi permet », mais « des amours sans risques » (Venerem tutam ${ }^{5}$ ). Nason ne cherche plus ici à séduire son lecteur en tenant un discours badin et pragmatique, voire cynique, mais à se défendre des attaques d'une censure envahissante en recourant à des arguments juridiques.

7 L'exilé renonce également à feindre la naïveté. Il n'ignore pas que l'interdit placé au début du recueil viendra plutôt stimuler la curiosité féminine et l'inviter à commettre une transgression érotique purement littéraire qui pourrait entraîner une transgression dans la réalité. Il assume cependant l'ambiguïté de l'instrument qu'il a choisi :

Persequar inferius, modo si licet ordine ferri,

Posse nocere animis carminis omne genus ;

Non tamen idcirco crimen liber omnis habebit :

Nil prodest quod non laedere possit idem 6 .

Cette concession faite aux auteurs qui, depuis Platon, en passant par Cicéron ${ }^{7}$, voyait dans la poésie "l'école du vice», prépare une riposte audacieuse: l'exilé démontre que l'ambivalence est propre à n'importe quel art ou science, et prend l'exemple de l'éloquence, qui devrait inspirer la même défiance que la poésie :

Discitur innocuas ut agat facundia causas :

Protegit haec sontes inmeritosque premit ${ }^{8}$.

Nason indique ainsi que ce n'est ni dans l'art, ni dans son objet (materia), que réside le mal, mais bien plutôt dans l'usage qu'en fait l'auteur ou celui auquel il destine son ouvrage, le lecteur. Dans la conception ovidienne, l'Amour apparaît comme une matière littéraire neutre, qui peut prendre n'importe quel visage sous le ciseau de son démiurge, l'auteur, ou sous celui du lecteur, qui poursuit le travail créateur sur les poèmes qu'il déchiffre. La parole est à moitié à celui qui la parle, à moitié à celui qui l'écoute, écrivait Michel de Montaigne. Nason est persuadé qu'un lecteur «à l'esprit droit » (recta (...) mente, vers 275) ne trouvera pas matière à critiquer ou à pécher dans ses vers. En revanche, la lectrice - ou le lecteur - que son tempérament entraîne à faillir (studiosa sinistri, vers 257, in culpam (...) ingeniosa suam, vers 288) y débusquera mille griefs, enseignements et incitations à mal faire.

10 Cet argument introduit un topos poétique, récurrent dans les Tristes, celui de l'Envie éprouvée par l'ennemi, qui prend ici le visage de l'interprète jaloux et calomnieux. C'est lui qui a nui au poète dans l'esprit du prince en lui soufflant une lecture perverse de ses œuvres (vers 211-240). Nason engage l'empereur à se démarquer de cette figure négative.

Nec tamen est facinus uersus euoluere molles;

Multa licet castae non facienda legant ${ }^{9}$.

11 Pour l'exilé, la lecture de poèmes sur l'amour n'est pas en soi un crime, elle est moralement indifférente ; c'est dans l'interprétation de cette lecture que peut se glisser la faute qui appartient alors tout entière au lecteur.

Cependant, puisque son art et sa matière sont ambigus et susceptibles de générer des interprétations tendancieuses, puisqu'en outre cette accusation d'immoralité, adressée à un livre, rejaillit sur l'auteur ${ }^{10}$, il revient au poète, comme à l'orateur, de manifester son ethos de uir bonus. Nason se plie à cet exercice dans les Tristes :

Crede mihi, distant mores a carmine nostro -

Vita uerecunda est Musa iocosa mea - 
Magnaque pars mendax operum est et ficta meorum :

Plus sibi permisit compositore suo ${ }^{11}$. l'auteur et le locuteur poétique, doit être examinée avec circonspection. Si Nason distingue ici clairement les deux instances, il ne cesse, à d'autres endroits, de tisser des liens entre ces deux identités, de les superposer sans jamais qu'elles ne se recouvrent totalement. En effet, le flou qui subsiste sur la sincérité des déclarations du locuteur élégiaque rend plus séduisante encore la lecture des recueils. Néanmoins cette affirmation montre que le sujet érotique présente au moins un avantage: dans un contexte de durcissement moral, il contraint le poète et le pouvoir à baisser le masque et à révéler quelle place est laissée à la fiction dans la littérature ; selon Ovide farouchement attaché à la liberté absolue du poète, l'imagination créatrice ne devrait pas connaître de limites. En distinguant explicitement l'auteur et le locuteur, Nason revendique auprès d'Auguste un droit à l'invention fictionnelle, même si, pour cela, le poète doit renoncer à son magistère moral : il ne cherche pas à éduquer les brus romaines (Non (...) Romanas erudiuntque nurus). Pour l'exilé, plus la législation morale est rigoureuse, plus la part laissée à la fiction en littérature devrait être grande, comme une bouffée d'air dans une atmosphère asphyxiante, surtout si son auteur renonce à adopter une posture didactique et ne représente donc plus la moindre menace.

Il commence par avouer ses regrets :

Nil nisi peccatum manifestaque culpa fatenda est :

Paenitet ingenii iudiciique mei $^{13}$.

16

Pourtant, après avoir imaginé de composer des vers plus conformes aux désirs de l'empereur, la rétractation ne tarde guère. L'exilé commence par montrer que les limites de son ingenium l'ont forcé à embrasser le genre mineur de la poésie érotique, retrouvant par là le motif de l'excusatio. Or, l'emploi de ce topos doit retenir notre attention, car, comme l'a montré $\mathrm{M}$. Labate ${ }^{14}$, il surgit rarement sous la plume d'Ovide, qui lui préfère la recusatio callimaquéenne. Dans les poèmes liminaires des Amours, contrairement à certains de ses prédécesseurs, Nason présente sa carrière élégiaque comme un choix délibéré et non comme un choix par défaut. Or, l'évocation, dans l'extrait des Tristes, du talent (ingenii) et du jugement (iudicii) de Nason nous semble une référence explicite à un texte important de Cicéron ${ }^{15}$ sur le choix d'un genre de vie et d'une carrière. Cette décision doit être prise en fonction de nos quatre personae déterminées par notre nature universelle d'être doué de raison, par notre nature singulière (natura ou ingenium), par les circonstances de notre existence et par notre propre volonté (uoluntas ou iudicium). L'aveu de Nason comporte deux des concepts cicéroniens et accorde, suivant en cela Cicéron, une place prépondérante à la uoluntas. Nous pensons en effet ${ }^{16}$ que, dans les Amours, Cupido, le Désir, qui transforme en distiques élégiaques les élans épiques de Nason, est un avatar de la uoluntas cicéronienne. Le jeune poète aurait le talent pour composer des épopées mais son cœur et son désir le portent ailleurs. Dans les Tristes, Ovide invoque donc son ingenium mais surtout sa uoluntas pour se justifier d'avoir suivi le camp d'Eros.

Nec liber indicium est animi, sed honesta uoluntas

Plurima mulcendis auribus apta ferens ${ }^{17}$. 
17 Outre le droit à la fiction, Nason demande celui de composer une œuvre qui ne revendique pas de visée didactique, du moins dans le domaine moral. Dans les Métamorphoses comme dans l'Art d'Aimer, en contant les amours incestueuses de Myrrha et de son père, il avait pris des précautions, en invitant les jeunes filles et les pères de famille à ne pas le lire,

Aut, mea si uestras mulcebunt carmina mentes,

Desit in hac mihi parte fides, nec credite factum ;

Vel, si credetis, facti quoque credite poenam ${ }^{18}$.

Ovide met ainsi en tension le pôle de la fiction plaisante et celui de l'enseignement moral en évoquant deux types de lecture sur cette aventure amoureuse : la lecture plaisante, « caressante ", ou la lecture morale. Lui-même cherche d'abord à plaire, à séduire avant d'instruire son lecteur. Mais il n'est pas non plus sans savoir, en employant le verbe mulcere, qu'en caressant les oreilles de ses lecteurs, il les façonne également et les transforme, comme, dans sa forge, Mulciber-Vulcain, l'époux jaloux et humilié de Vénus, qui ne peut se passer d'elle tout en haïssant les humiliations qu'elle lui impose : l'Art d'Aimer et les Remèdes à l'amour enseignent à trouver du plaisir dans l'amour, et à se débarrasser de la souffrance, éventuellement en trouvant du plaisir jusque dans la souffrance ${ }^{19}$. L'idéal esthétique se substitue à l'idéal éthique ou, plutôt, la matière érotique permet à Ovide de réordonner les pôles du docere et du placere en forgeant une nouvelle éthique, celle du plaisir, comme le souligne l'emprunt de l'expression honesta uoluntas au discours moral et philosophique.

Nous conclurons cette première partie de l'analyse en soulignant que Nason corrèle sa relation à l'écriture, sa materia et l'effet produit sur le lecteur. Puisque c'est le Désir qui l'amène à composer des élégies, il marque nécessairement ses œuvres. Cette empreinte est formelle, puisque Cupido ôte un pied à ses vers épiques et crée un "vide», un déséquilibre qui mime l'élan du désir alimenté par l'absence de l'objet désiré, mais elle est aussi thématique, puisque Cupido devient le sujet bien-aimé de l'Élégie avec laquelle il a des affinités :

Sum leuis, et mecum leuis est, mea cura, Cupido ;

Non sum materia fortior ipsa mea ${ }^{20}$.

La lasciuia, le "libertinage ", la « licence ", symbolise cette continuité entre la matière érotique et la forme poétique. L'adjectif lasciuus est régulièrement une épithète de l'Amour ovidien mais revient aussi sous la plume de Quintilien, qui caractérise par cet adjectif péjoratif le style d'Ovide, à qui il reproche également d'être «trop attaché à son génie » (nimium amator ingenii sui ${ }^{21}$ ). La lasciuia unit dangereusement les domaines de la moralité et de la stylistique, au point de déchaîner le courroux augustéen, incapable de distinguer les niveaux de la fiction littéraire et de la réalité.

Haec tibi me inuisum lasciuia fecit ob Artes

Quas ratus es uetitos sollicitasse toros ${ }^{22}$.

21 La lasciuia désigne le libertinage érotique, mais aussi rhétorique, qui entretient savamment la confusion entre l'expérience et la fiction pour le plus grand plaisir de l'auteur et de son lecteur, gagné par le désir de lire et d'aimer autant les puellae que les carmina du poète. Nason rappelle alors opportunément, sans hésiter à gauchir l'œuvre de ses prédécesseurs, que, quel que soit le genre dans lequel ils aient choisi de s'illustrer, l'Amour constitue le noyau autour duquel Homère, les Tragiques grecs, Ennius, Lucrèce ou même Virgile, ont élaboré une intrigue et une esthétique afin de captiver leurs lecteurs. C'est justement la lasciuia de Cupido qui lui permet de déborder les cadres 
génériques bien définis et les mesures (modi) qu'on tente de lui imposer. Comme Dédale ${ }^{23}$, quand on tente de lui barrer une route, il réussit à s'élever et à se métamorphoser pour franchir les obstacles qu'on lui impose.

Il usera de ce don dans toute l'œuvre ovidienne. Dans les Fastes, Ovide rend hommage à Vénus et l'assure que loin de l'avoir abandonnée, il a décidé de la servir autrement en vieillissant ${ }^{24}$. Par la suite, dans les Tristes et les Pontiques, puisque l'exil et son âge lui interdisent d'évoquer son désir pour une puella, Nason remodèle le visage de Cupido qui lui apparaît une nuit, la mine abattue et le corps épuisée ${ }^{25}$, pour lui donner les traits sombres du desiderium, le «désir nostalgique » que lui inspirent désormais des objets convenant à son âge et à son ethos de poète repenti. Les recueils d'exil chantent ainsi sa patrie, son épouse, ses amis, mais aussi la poésie élégiaque qu'il ne cesse jamais de chérir.

Sentit amans sua damna fere, tamen haeret in illis,

Materiam culpae persequiturque suae.

Nos quoque delectant, quamuis nocuere, libelli,

Quodque mihi telum uulnera fecit, amo ${ }^{26}$.

Le poète fait de la poésie son ultime passion, le désir que rien ne pourra venir satisfaire et dont il ne pourra se défaire sans perdre la vie.

En conclusion, nous pourrions dire que, pour Ovide, l'Amour, précisément en raison des effluves sulfureux du libertinage, demeure un indice de la liberté laissée aux auteurs littéraires de développer des récits fictionnels et audacieux, dans un contexte de durcissement moral, sans avoir nécessairement de visée didactique éthique. Eros-Cupido constitue en outre chez Ovide le fondement d'une poétique du désir, qui le prend pour sujet (materia) mais aussi pour référence stylistique, puisque sa plasticité et sa lasciuia, sa liberté absolue et irréductible, lui donnent la capacité de se métamorphoser sans cesse pour inspirer des poèmes de tous les genres et dans tous les mètres possibles.

\section{Melissus ou la croyance en la fécondité d'Amour en poésie}

Il semble intéressant d'essayer de mesurer comment cette défense du sujet érotique en poésie a été reçue et exploitée chez le poète protestant Melissus. Ce dernier a bien perçu l'importance du concept de lasciuia, puisqu'il en fait le cœur de la première concession faite aux censeurs de la poésie érotique antique à la Renaissance.

Multa quidem pro more satis lusere licenter;

Lasciuitque animi luxuriantis opus ${ }^{27}$.

Melissus se garde bien de lever sur ce travers un sourcil sévère. Si l'insistance sur la liberté (licenter, lasciuit) montre qu'il a parfaitement compris l'enjeu du débat, il préfère diminuer la portée subversive de la lasciuia, agent de confusion et de transgression, en recourant à l'expression relativisante pro more et en la rapprochant du jeu (lusere), du badinage textuel que ne peuvent réfréner les poètes quand ils lâchent la bride à leur inventivité. Par là, il affaiblit sans doute la portée et la puissance poétique de ce concept éthique et rhétorique. Cependant, la neutralisation de la lasciuia lui permet de sauver la lecture de la poésie antique en réduisant ses effets à une pure question de forme ou d'expression: si quelques termes obscènes surgissent ici et là, il est aisé d'en faire abstraction pour se concentrer sur le fond, beaucoup moins audacieux qu'il n'y paraît.

Excipias obscena; pudens nihil auris habebit

Offensam quo se possit anile queri ${ }^{28}$. 
Pour Melissus, il ne s'agit que d'une affaire de contexte: il affirme qu'il y a identité de nature entre l'amour illégitime chanté par les poètes antiques et l'amour conjugal prôné par la morale protestante.

Finge ea, quae paullo tibi liberiora uidentur,

Dicier in castis ingenuisque toris :

Ecquid in his uitii est ? Quasi uero nulla Pudori,

Nulla uerecundis sit data causa Iocis!

Turpius interdum dictu factuque maritis

Quiddam usu, ueteres quam meminere, venit.

At uero non id uulgandum ${ }^{29}$.(...)

Pour opérer cette transposition, l'auteur allemand s'appuie sur une caractéristique de la poésie élégiaque antique ${ }^{30}:$ loin de rejeter entièrement les valeurs anciennes de Rome, les élégiaques ont choisi de transférer certaines des vertus politiques traditionnelles comme la fides, le pudor, la pietas et la uerecundia - dans le domaine de la relation érotique illégitime. Les poètes élégiaques montrent parfois plus de respect et de dévouement à leur maîtresse que nombre de Romains ou de Protestants de l'époque de Melissus en témoignent à leur épouse ou même à leur patrie.

Pour rendre moins sulfureuse la fréquentation de ces poètes, Melissus n'hésite pas à les dévêtir, en quelque sorte, de leurs pointes audacieuses ou, plutôt, à encourager une «translation contextuelle », en quelque sorte. Il rejoint ainsi les pratiques de la lecture allégorique que le Moyen-Âge et la Renaissance avaient déployé sur les textes antiques afin de concilier leur contenu avec la doctrine chrétienne. Il ne faudrait pas réduire le projet de Melissus à des astuces : il s'emploie à défendre la poésie érotique antique en élaborant une véritable casuistique. Il est ainsi possible de discerner, derrière cette transposition de la relation illégitime à la relation conjugale, l'influence de la doctrine érasmienne de l'aptum rhétorique, développée notamment dans le Ciceronianus (1528). Érasme s'en prenait avec véhémence à ceux qui prétendaient figer le latin dans son usage antique. Au contraire, soulignant les disparités morales, religieuses, politiques ou économiques entre l'Antiquité et la Renaissance, et en particulier le fossé creusé par la révélation chrétienne, il incitait ses lecteurs, dans leur pratique de la langue latine, à prendre en compte les innovations et les bouleversements apportés par l'histoire et les circonstances contemporaines. Implicitement, Melissus ne propose rien d'autre que d'appliquer ce principe de l'aptum à la lecture, qui doit être elle aussi ancrée dans l'ici et maintenant, adaptée au contexte contemporain du lecteur afin d'en «neutraliser » les incongruités. Le lecteur humaniste ne doit pas s'arrêter à la lettre des poèmes, qui pourrait heurter, mais plutôt les étudier en profondeur, pour y découvrir le caractère universel de certains sentiments ou expériences qui confèrent à ces poèmes leur intérêt.

(...) Quid, quod et illi

Verbis ficta nothis et simulata canunt?

Scilicet ut prosint, ut delectentque legendo.

Sis bonus interpres, nil erit inde mali ${ }^{31}$.

L'attaque des détracteurs de la poésie antique paraît se concentrer, après les reproches sur le caractère licencieux de leur poésie, sur leur recours à une langue sophistiquée et sur leur attrait pour l'invention fictionnelle, revendiquée par Nason. Pour les défendre, Melissus invoque lui aussi la dialectique complexe qui doit s'instaurer entre la visée didactique et la visée esthétique. Avec prudence, il choisit cependant de mentionner l'utile avant le dulce. L'argument de la lecture allégorique est judicieusement employé, puisque l'allégorie est vantée pour son efficacité pédagogique : elle permet de dissimuler 
un enseignement un peu rebutant sous un discours plaisant. Les deux fonctions de la littérature, l'utile et l'agréable, sont ainsi harmonieusement réunies. Il est particulièrement intéressant de remarquer que le recours à l'allégorie et à la lecture « recontextualisée » font du lecteur melissien, comme du lecteur ovidien, un interpres, responsable du sens qu'il donne au texte. L'influence du protestantisme, qui incite chacun à lire le texte de la Bible et à en construire le sens, sans médiation, facilite la référence à cette figure du lecteur-interprète. Ainsi, comme dans les Tristes, c'est l'état d'esprit du lecteur qui donnera au texte son sens moral ;

Obuia sunt ueterum, sunt obuia multa recentum:

Mentem adhibe castam, crimine lingua uacat ${ }^{32}$.

31 L'allégorie, souvent utilisée par les auteurs chrétiens pour rendre acceptables les textes païens, induit une pratique de l'exégèse qui permet d'articuler des mondes différents. C'est au nom d'une religion dans laquelle la transcendance est essentielle que Melissus réinterprète une poésie païenne écrite à un moment où un seul homme, Auguste, cumulait les pouvoirs politique, religieux et culturel : c'était par rapport à cette figure qu'Ovide devait plaider sa cause et mobiliser ses talents rhétoriques. En se plaçant dans la perspective d'une spiritualité supérieure, autrement dit en relativisant les jugements humains, Melissus transforme le plaidoyer en arme offensive contre tous ceux qui enferment le sens dans les limites nécessairement trop étroites de la littérarité des mots. Les vitupérations contre le mauvais lecteur apparaissent à la fin de l'élégie de Melissus, corrélées au topos de l'envie qui s'acharne sur les écrits des poètes brillants. Il conclut assez sévèrement :

Stultorum nemo sapit, ecquid carminis artem,

Aut ipsos deceat carminis artifices ${ }^{33}$.

Il atteint ainsi le cœur de son argumentation en tentant de cerner le devoir (decorum) du poète. Le poète allemand cite l'exemple de Veselius, alias Théodore de Bèze, natif de Vézelay : avant de se consacrer à des œuvres plus sérieuses quand il s'installa à Genève, il s'était essayé, dans ses Juuenilia, à la poésie érotique qui lui attira les foudres de certains censeurs :

(...) Sic oblectamine salso

Ludicra uulgavit carmina Veselius ;

Candida cui stimulum uirtutis ad optima quaeque,

Applausum Phoebo non renuente, dedit ${ }^{34}$.

L'exemple particulier de Théodore de Bèze permet à Melissus d'illustrer ce qui, selon lui, fait la grandeur du rôle des poètes: le vide, l'aspiration créé au départ par l'aiguillon d'Eros peut se révéler une propédeutique à la conception de pensées, de désirs ou d'œuvres plus élevées. L'Amour humain et l'Amour divin découlent en effet de la même source. Si l'Amour humain peut dégénérer au point de devenir méconnaissable, il peut également se sublimer en élan vers la divinité : cette conception de l'Amour, défendue notamment par le néo-platonicien florentin Marsile Ficin ${ }^{35}$, séduisit nombre d'artistes de la Renaissance qui ne devaient pas manquer de souligner la parenté entre la pulsion érotique et la pulsion artistique, toutes deux provoquées par le désir de contempler sans fin un objet dont l'artiste s'emploie à reproduire dans le sensible l'image devenue intelligible par l'intériorisation. Melissus relie lui aussi ces deux élans en décrivant le mécanisme du désir poétique, stimulé et gouverné par les "simulacres» de la femme aimée.

Nos quoque (si numero uatum non eximor, Hagi,

Si qua meis uis est ingeniosa libris) 
Speratam numeris fictis cantare Rosinam,

Per bis terna fere lustra peracta, iuuat.

Illam ego nec uisam, nec posthac forte uidendam

Depereo, miserum tostus amore iecur.

Illam ego per sentes aspros, et inhospita tesqua,

Exterus ignotis aduena quaero locis.

Nullus ad arbitrium uersus mihi, nulla sine illa,

Tunc quoque cum meditor seria, Musa uenit.

Siue diem caelo Matuta reuelet aperto,

Seu fuscus gelidam Vesper obumbret humum,

Illius optatos suspiro cernere uultus,

Quos Morpheus oculis subdidit ante meis :

Iucundisque inhians simulacra retecta figuris,

Materiae ductum, corpus ut umbra, sequor ${ }^{36}$.

Melissus relate que c'est le désir érotique qui a déclenché chez lui une quête poétique. À l'instar des poètes élégiaques et de Pétrarque, Melissus fait de la femme désirée son unique Muse, qui l'empêche de composer des ouvrages plus sérieux, et qui l'attache à ces illusions qu'il poursuit. En revanche, contrairement à ces prédécesseurs, il prend soin de révéler ici très vite ce qu'Ovide ne consentait à avouer que dans les Tristes: la femme aimée n'existe pas, elle a été forgée par l'imagination du poète pour lui inspirer ces rythmes auxquels elle confère son caractère fictif (numeris fictis). Elle n'a jamais eu d'existence sensible, elle appartient au monde intelligible mais elle n'est pas pour autant dépouillée de toute corporéité : elle est une image, un "simulacre», cette pellicule atomique qu'émettent les corps dans la pensée lucrétienne; à cette image, le poètePygmalion sculpte peu à peu un corps de mots. C'est le désir érotique et artistique du poète qui a transformé en matière cette femme de rêve. Au lieu de transformer une femme réelle en figure littéraire, le désir du poète a fait advenir à l'existence une image conçue dans le secret de son cœur.

Melissus prend ainsi ses distances avec la pensée lucrétienne de l'amour ${ }^{37}$, largement relayée par $\mathrm{M}$. Ficin ${ }^{38}$. Pour Lucrèce en effet, la pulsion érotique est purement physiologique et préexiste à la rencontre d'un homme ou d'une femme dont l'amoureux va progressivement se persuader qu'il est le ou la seul(e) à pouvoir l'apaiser. Il n'est pourtant captif que de simulacres et d'un désir qui ne peut être satisfait, même par l'étreinte. Pour éviter de se consumer dans la passion suscitée par ces simulacres, il convient alors de se détourner des images qui alimentent la passion (pabula amoris) et de déverser l'humeur virile dans le corps de la première femme venue. Melissus emprunte une toute autre voie, dans le prolongement de la pensée ovidienne ${ }^{39}$, puisqu'il considère au contraire qu'il faut au contraire construire un objet spécifique à cette pulsion préexistant afin d'exacerber le désir érotique jusqu'à lui donner une fécondité artistique. Il cite ainsi l'exemple du poète Lotichius chez qui le désir de composer des vers élégiaques existait avant même qu'il eût une femme pour les inspirer. C'est Erato, la muse de la poésie érotique, qui, comme Cupido dans les élégies I, 1 et 3 des Amours, donne au poète un objet digne de ses vers :

Lotichius libuit priscorum imitarier umbras,

Nec canere imparibus carmen inane sonis.

Mollia quo melius fluerent in carmina uenae,

Naturaeque opulens ars adhiberet opem;

Auribus et doctis possent tot scripta probari,

Gratiaque argutis maior inesse modis: 
Purpuream faculis Erato iucunda puellam

Obiecit, dignam uatis amore $\operatorname{sacri}^{40}$. humaniste et protestant, il ne faut pas se résigner à satisfaire ce désir en s'abandonnant à une étreinte dégradante ou à la composition de vers obscènes. Au contraire, il convient de continuer à le nourrir en s'adonnant à la contemplation des simulacres pour s'élever jusqu'à la source spécifique, qui n'est ni celle de la poésie basse et obscène ni celle de la poésie épique, religieuse ou philosophique, à laquelle l'élégie puise son badinage :

Non mihi propositum est lasciuo fallere lusu

Tempus, et impuro deterere ora sale.

Odi ego Lampsacios, puerorum incommoda, versus :

Casta uerecundae carmina mentis amo.

Attamen innocuae mellita reperta Camenae,

Quaeque Amor insomni nocte iterare iubet,

Cur tendam sepelire prius, quam nata videntur,

Aut Genio fraudes arte parare uafra?

Quin potius documenta suae scitissima formae

Promere sub lucem daedala Pallas amet.

Haec suscepta mei sunt argumenta laboris ;

His uagus erro diu ludor imaginibus :

Forsan et his alii ludentur; uera putantes,

Quae speciem ueri decipientis habent.

Credula res, nugae ${ }^{41}$. (...) la vérité : si les aventures érotiques relatées par ses vers n'ont pas de réalité, puisqu'elles ne sont qu'images suscitées par une imagination débordante, elles ont une vérité du sentiment et une vraisemblance, qui impose au poète humaniste de les plier aux impératifs de la morale contemporaine. En effet, contrairement à Ovide, Melissus prend nettement ses distances avec les excès du libertinage dans les quatre premiers vers de ce passage, sans pour autant consentir à renoncer à l'inspiration d'Eros.

L'Amour mélissien est en effet fortement moralisé. Modelé sur l'Eros élaboré par la pensée néo-platonicienne, il descend du Ciel :

(...) cui fronte Modestia recta

It comes, et niveo corde pudica Venus.

Purior hunc Aether olim generavit, et intra

Succensas iussit regna tenere fibras ${ }^{42}$.

Cette origine et ces compagnes lui imposent des bornes strictes pour qu'il conserve une puissance à laquelle l'univers ne peut choisir que de céder ou de contribuer (vers 31-50) : le désir érotique du poète doit se plier au cadre conjugal ou demeurer dans une tension perpétuelle, dans une avidité désirante jamais assouvie, pour devenir fécond. En effet, au thème de l'avidité, de la soif et du désir impossibles à satisfaire, répondent en effet ceux de la nourriture, de l'aliment et du breuvage qu'Amour prodigue à ses adorateurs. Une autre image lucrétienne ${ }^{43}$ transparaît en filigrane, quand Melissus représente l'Amour instillant goutte-à-goutte une substance dans le cœur de l'homme :

Si quid in humano genere est, procliuius ad se

Mentes blanditis quod trahat illiciis,

Vimque suam instillet totis Geniumque medullis,

Iugeque inescato pectore nectar alat

Certe Amor est, Hagi ; (... $)^{44}$. 
Chelissus cependant, l'Amour est plus nourrissant et plus bienfaisant que chez Lucrèce. Il emplit le cœur de l'homme non « d'une froide passion » (frigida cura) mais de puissance et de génie. Le mot Genius unit les fils de la tradition philosophique du daimôn à la thématique de la fécondation qui fonde le rôle d'Amour dans l'univers. En proposant un récit des premiers temps de l'humanité, inspiré lui aussi de la genèse néo-platonicienne ${ }^{45}$, Melissus fait de l'Amour une force qui unit l'homme à la femme et féconde le couple en lui donnant des enfants, avant d'essaimer dans tous les domaines, comme le Désir ovidien, en attachant à leur foyer les hommes et en les reliant entre eux par les liens de l'amitié : l'amour et l'amitié, exprimés tous deux en latin par le terme amor, sont pour Melissus de même nature.

Quand la pulsion érotique n'est pas satisfaite dans un cadre conjugal, politique ou économique, quand elle demeure dans une tension désirante sans être assouvie, loin de devenir stérile, elle éveille le génie des érudits et crée entre eux une solidarité amicale et féconde. Joignant l'exemple à la théorie, Melissus fait apparaître une chaîne de poètes dont le génie créateur a été révélé par l'Amour; il unit les auteurs antiques d'élégies érotiques (Catulle, Ovide, Properce et Tibulle, auxquels il adjoint le Virgile des Bucoliques, Gallus, Cinna, Calvus et Varron) à Pétrarque, à Lotichius et enfin à lui-même. Quoiqu'elle se fasse plus discrète au centre du poème, elle passe aussi par Théodore de Bèze, que l'auteur de l'élégie défend, avant que Melissus n'évoque ses contemporains et, implicitement, les générations à venir: Scaliger, Dousa, Lernutus; la troupe zélée ( studiosa) des poètes connaît son devoir : défendre les poètes érotiques contre la hargne des censeurs. Melissus réalise ainsi ce qui était un vœu pieux de la poésie d'exil ovidienne, la solidarité entre tous les poètes, quel que soit le genre dans lequel ils aient choisi d'exercer leur talent, au nom de leur passion (studium) commune pour les belles-lettres. distiques de la pièce, à l'image d'un Amour nourricier qu'il représente non plus penché sur la race humaine toute entière, mais sur les poètes et leurs lecteurs. S'étant substitué aux Muses, il les nourrit non plus de nectar, mais de ce miel dont Melissus, poète de l'Amour, a pris le nom.

Nam quia gustarunt semel intemerata mearum

Munera, quae succo roris aluntur, apum,

Nescio qua genialis Amor melligine uates

Lactat, et oblitos non sinit esse faui ${ }^{46}$.

L'Amour chanté par Melissus à la Renaissance est ainsi fortement moralisé, contrairement à celui de l'élégie érotique antique, qui doit être "relue» et « recontextualisée » pour être sauvée. Paradoxalement, l'amour élégiaque apparaît alors, du point de vue de l'axiologie, comme un moment intermédiaire entre le mos maiorum et le christianisme. L'amour ne pouvait pas se dégager immédiatement, dans sa pureté spirituelle, des valeurs païennes. Il fallait qu'il apparût d'abord lié au système de valeurs antérieur avant de s'épurer en éthique chrétienne du mariage. De ce fait, la poésie élégiaque est justiciable d'une double approche. L'une critique, que Melissus récuse. L'autre, sensible au mouvement des idées, qui permet à Melissus son audacieuse relecture. À cette époque et dans l'Allemagne protestante, le désir érotique ne se sépare pas de la Vénus pudique, gouverne les lits conjugaux et prodigue sa force au poète à la seule condition de ne pas être épuisé dans les étreintes avec une femme réelle. Si Melissus distingue bien, comme Ovide, le plan de la fiction, du rêve, et celui de l'expérience, ce n'est pas, cependant, le seul fondement de sa défense de l'Amour ; même dans la fiction, à 
cette époque, Eros doit conserver ses barrières morales. En revanche, les deux poètes se retrouvent quand ils vantent la puissance créatrice de l'Amour, capable de révéler le génie littéraire de ceux qu'il touche de son aiguillon:

Fallimur, an teneris anima est Amor ipse Camenis, Viuacisque illex proditor ingenii ${ }^{47}$ ?

C'est au nom de cette fécondité artistique du sujet érotique que les deux poètes défendent Eros en littérature, refusant que les censeurs brident leur inventivité et préférant s'en remettre au bon lecteur. C'est à lui qu'ils confient les fruits d'un talent (ingenium) porté à son incandescence par l'amour : à son tour, le lecteur saura déployer sur leurs vers les talents d'interprète que confère l'amour de la littérature et de ses auteurs.

\section{BIBLIOGRAPHIE}

LABATE Mario, L'arte di farsi amare, Modelli culturali e progetto didascalico nell'elegia ovidiana, Pisa, Giardini, 1984.

LEVY Carlos, « Aimer et souffrir : quelques réflexions sur la «Philosophie dans le boudoir » de l'Ars amatoria » dans C. Lévy et L. Boulègue (dir.), Hédonismes. Penser et dire le plaisir dans l'Antiquité et à la Renaissance, Villeneuve d'Ascq, Presses Universitaires du Septentrion, 2007, p. 161-172.

NERAUDAU Jean-Pierre, «Ovide ou la difficulté d'être un auteur, Réflexions sur les Tristes et les Pontiques », dans G. Chamarat et A. Goulet (dir.), L'auteur, Colloque de Cerisy-la Salle (4-8 Octobre 1995), Caen, Presses Universitaires de Caen, 1996, p. 27-35.

NILGES Annemarie, Imitation als Dialog : die europäische Rezeption Ronsards in Renaissance und Frühbarock, Heidelberg, C. Winter, 1988, p. 43-64.

SCHAEFER, Eckart, « Paul Schede Melissus », dans S. Füssel (dir.), Deutsche Dichter der frühen Neuzeit (1450-1600). Ihr Leben und Werk, Berlin, E. Schmidt, 1993, p. 545-560.

SCHAEFER, Eckart, « Lotichius' Liebesdichtung - ein Experiment mit dem Leben », dans U. Auhagen et E. Schäfer (dir.), Lotichius und die römische Elegie, Tübingen, Gunter Narr Verlag, 2001, p. 241-295.

LUDWIG Walther, « Un traité protestant sur le mariage - le recueil des Carmina et epistola de coniugio ad D. Davidem Chytraeum (1562) », dans P. Galand-Hallyn et J. Nassichuk (dir.), Le lyrisme conjugal à la Renaissance, à paraître à Genève, Droz, 2009.

Je tiens à remercier le professeur C. Lévy dont les suggestions judicieuses ont enrichi cette étude.

\section{NOTES}

1. Ovide, Tristes, II, 211-212. Nous citons le texte établi par J. André dans la Collection des Universités de France, 1968. Les traductions sont nôtres. 
2. Paul Schede Melissus, Nuremberg, 1583 puis Élégies, IV, 2, p. 107-114 dans Schediasmata Poetica; secundo edita multo auctiora, Paris, Sittart, II, 1586 : «À Johannès Hagen. L'Amour a été inspiré par les dieux aux hommes dès leur origine, aussi les poètes ne cessent de le chanter dans leurs écrits. Défense de Lotichius et de l'auteur. » Toutes les citations de Melissus seront tirées de cette élégie. Cette pièce est lisible sur le site internet http://www.uni-mannheim.de/mateo/camena/ melis1/books/schedeg_2-4.html. E. Schäfer, dans son article, «Lotichius' Liebesdichtung - ein Experiment mit dem Leben », dans U. Auhagen et E. Schäfer (éds.), Lotichius und die römische Elegie, Tübingen, Gunter Narr Verlag, 2001, p. 241-295 a étudié l'image de Lotichius construite par Melissus dans cette élégie, p. 284 et suivantes.

3. Ovide, Tristes, II, 243.

4. Ovide, Tristes, II, 249-250 : «Dans nos chants, rien que ce que la loi permet, rien que des larcins licites,/ dans mon poème, rien n'encourra d'accusation. »

5. Ovide, Art d'aimer, I, 33.

6. Ovide, Tristes, II, 263-266 : «J'exposerai plus loin, si seulement je puis traiter mes arguments point par point/ que toute sorte de poésie peut mettre les esprits en péril ;/ Ce n'est pas pour autant que tout livre encourra une accusation:/ Il n'y a rien de profitable qui ne puisse aussi faire du tort. »

7. Cicéron, Tusculanes, IV, 69.

8. Ovide, Tristes, II, 273-274 : «On apprend à bien parler pour plaider la cause des innocents:/ L'éloquence sauvegarde les coupables et s'acharne sur ceux qui n'ont rien fait. »

9. Ovide, Tristes, II, 307-308 : «Nul crime, cependant, à parcourir des vers voluptueux ;/ La femme vertueuse peut lire bien des choses qu'elle n'a pas le droit de faire. »

10. Sur la confusion entre l'auteur et le locuteur de l'Art d'aimer que Nason reproche implicitement à Auguste, voir J.P. Néraudau 1996, p. 27-35.

11. Ovide, Tristes, II, 353-356 : «Crois-moi, mes mœurs sont loin d'imiter ma poésie -/ ma vie est respectable, tandis que ma Muse est badine !-/ Mes œuvres sont pour l'essentiel mensonges et inventions :/ elles se sont permis bien plus qu'à leur auteur. »

12. Ovide, Tristes, II, 313-314 : « Mais pourquoi notre Muse est-elle excessivement licencieuse,/ et pourquoi mon livre conseille-t-il à chacun d'aimer?»

13. Ovide, Tristes, II, 315-316: "C'est une erreur et même, il me faut le reconnaître, une faute évidente:/je me repens de mon talent et de mon jugement. »

14. M. Labate, L'arte di farsi amare, Modelli culturali e progetto didascalico nell'elegia ovidiana, Pisa, Giardini, 1984, p. 26.

15. Cicéron, De officiis, I, 107-115.

16. Sur ce point, voir notre thèse, Sed desiderium superest, Poétique de la nostalgie dans les élégies d'exil d'Ovide et les Elegiae de Petrus Lotichius Secundus (1528-1560), à paraître à Genève, chez Droz.

17. Ovide, Tristes, II, 357-358 : «Mon livre ne vient pas dévoiler mon cœur, mais il est guidé par l'honorable volonté/ d'offrir cent douceurs venant caresser les oreilles. »

18. Ovide, Métamorphoses, $\mathrm{X}, 301-303$ : « Ou, si mes vers viennent caresser vos cœurs,/ n'accordez pas foi à ce que je relate ici, ne croyez pas au crime ;/ ou bien, si vous y croyez, croyez aussi au châtiment qui s'ensuivit. »

19. Sur l'étroite connexion entre le plaisir et la souffrance dans l'Art d'aimer, voir C. Lévy 2007, p. 161-172 et plus particulièrement $\mathrm{p}$. 168-171.

20. Ovide, Amours, III, 1, 41-42 : « Je suis légère, et, comme moi, léger est mon souci, Cupidon./ Je n'ai moi-même pas plus de force que mon sujet. »

21. Quintilien, Institution Oratoire, $\mathrm{X}, 1,88$.

22. Ovide, Tristes, II, 345-346: «La licence que j'ai prise dans mon Art m'a rendu haïssable à tes yeux,/ tu as cru qu'il cherchait à séduire des lits défendus. »

23. La comparaison se trouve dans l'Art d'aimer, II, 15-98.

24. Ovide, Fastes, IV, 3-10. 
25. Cette apparition est relatée dans les Pontiques, III, 3.

26. Ovide, Tristes, IV, 1, 33-36 : «En général, un amant sent qu'il court à sa perte, cependant, il s'y attache,/ et poursuit l'objet de sa faute./ Je leur trouve aussi bien des charmes, même s'ils m'ont été fatals, à mes petits livres ;/ le trait qui a causé ma blessure, je l'aime. »

27. P. Schede Melissus, 55-56: «Certes, par rapport à l'usage, ils badinèrent souvent, avec une certaine liberté ;/ et l'œuvre de leur esprit débordant fut licencieuse. »

28. P. Schede Melissus, 57-58 : «Retires-en les obscénités, une oreille pudique ne trouvera rien/ qui lui permette de gémir comme une vieille d'en être offensée. »

29. P. Schede Melissus, 59-65: «Imagine que les propos qui te paraissent un peu trop libres/ soient prononcés dans des lits vertueux et bien nés./ Leur trouves-tu un caractère vicieux? Comme si jamais à la vraie Pudeur,/ Aux Plaisanteries respectueuses on ne donnait la parole !/ Parfois, il arrive aux époux d'évoquer ou d'accomplir un acte plus honteux/ Que ceux relatés par les Anciens./ Mais il convient de n'en point parler.»

30. Voir, sur ce sujet, J. P. Boucher, Études sur Properce. Problèmes d'inspiration et d'art, Paris, de Boccard, 1965 qui consacre un long chapitre au statut de la fides chez Properce, p. 85-105 ; S. Lilja, The roman elegists' attitude to women, Helsinki, Suomalainen Tiedeakatemia, 1965, p. 226-238 et G. B. Conte, «L'amore senza elegia. I Rimedi contro l'amore e la logica di un genere ", Generi e lettori, Lucrezio, l'elegia d'amore, l'enciclopedia di Plinio, Milano, Arnoldo Mondadori Editore, 1991, p. 53-94 et en particulier p. 58.

31. P. Schede Melissus, 65-68 : «(...) Que dire encore du fait/ qu'ils chantent en empruntant des termes étrangers des aventures qu'ils ont feintes et forgées?/ Naturellement, c'est afin qu'on trouve profit et plaisir à la lecture./Interprète-les bien et il n'en sortira rien de mal. »

32. P. Schede Melissus, 69-70: "Nous avons à notre disposition beaucoup d'ouvrages des anciens, beaucoup des modernes,/ Aborde-les avec un cœur vertueux : leur langue est dénuée de crime. »

33. P. Schede Melissus, 207-208: «Aucun de ces sots personnage ne sait quel devoir incombe à l'art de la poésie/ ou même aux artistes de la poésie.»

34. P. Schede Melissus, 193-196: « (...) Ainsi, mettant un peu de sel dans ses divertissements,/ Le Vézélien a publié des vers badins ;/ Candida lui a pourtant donné la vertu qui l'a aiguillonné vers les meilleurs sentiments,/ Sans que Phœbus ne lui refuse ses suffrages. " Candida était le pseudonyme que Théodore de Bèze donna à la bien-aimée chantée dans ses vers de jeunesse.

35. M. Ficin, Commentaire sur Le Banquet de Platon, De L'Amour, texte établi, traduit, présenté et annoté par P. Laurens, Paris, Les Belles Lettres, 2002, II, 7, p. 38-43.

36. P. Schede Melissus, 153-178: «Moi aussi (si l'on ne me retranche pas de la troupe des poètes, Hagen,/ s'il y a quelque puissance d'invention dans mes ouvrages)/ j'ai plaisir à chanter sur des rythmes de fiction Rosine que j'ai espérée,/ pendant que s'écoulaient deux fois trois lustres./ Moi, pour elle que je n'ai jamais vue, que jamais peut-être je ne verrai,/ je ne meurs pas, consumé d'amour jusqu'au fond de mon cœur malheureux./ Mais moi cette femme, par les sentes escarpées, par les déserts inhospitaliers,/ je la poursuis, en étranger venu de loin dans des lieux inconnus./ Nul vers ne me vient selon mon bon plaisir, nulle Muse sans elle,/ même quand je médite des poèmes sérieux./ Que l'Aurore dévoile le jour dans les nues qui se découvrent,/ ou que le Soir sombre enténèbre le sol glacé,/ j'aspire à contempler son visage désiré,/ que Morphée a offert jadis à mes regards:/ et attiré par les simulacres que m'ont dévoilés des figures agréables,/ Je suis la matière qui me guide, comme l'ombre suit le corps. »

37. Les développements sur le rôle des simulacres dans l'amour se trouvent au livre IV du De rerum natura, entre les vers 1030 et 1287.

38. M. Ficin, Commentaire sur Le Banquet..., VII, 5-11, 222-237.

39. Sur le dialogue d'Ovide avec la pensée lucrétienne, dont il réfute implicitement les conclusions et les recommandations dans l'Art d'aimer, voir C. Lévy, Aimer et souffrir..., p. 163-169. 
40. P. Schede Melissus, 93-100 : «Lotichius désira suivre les ombres des Anciens,/ sans entonner un poème vain aux accents inégaux./ Pour que sa veine s'écoulât plus aisément dans ses tendres poèmes,/ pour que l'art généreux vînt soutenir la Nature,/ pour que des écrits, si nombreux, pussent agréer aux doctes oreilles,/ et pour qu'on tînt en plus haute faveur ces mesures mélodieuses,/ Erato la charmante, de ses traits, mit sous ses yeux une beauté lumineuse,/ Digne de l'amour du poète sacré. "

41. P. Schede Melissus, 179-192:«Je n'ai pas pour dessein de tromper les heures dans un jeu licencieux,/ et d'user ma bouche par des traits impurs./ Moi, je hais les vers de Lampsaque, mauvaise habitude des jeunes gens,/ et ce sont les poèmes vertueux d'un esprit respectueux que j'aime./ Cependant, les trouvailles melliflues d'une Camène innocente,/ et celles qu'Amour nous fait répéter dans les nuits sans sommeil,/ Pourquoi m'efforcerais-je de les ensevelir avant même qu'elles paraissent au jour,/ ou de tendre des pièges à mon Génie en déployant un art subtil ?/ Les exemples les plus raffinés de sa beauté/ Puisse plutôt l'ingénieuse Pallas prendre plaisir à les mettre en lumière./ Telles sont les entreprises qui fondent mon travail:/ Je vagabonde et j'erre longtemps, en laissant ces images se jouer de moi./ Peut-être que d'autres en seront aussi les jouets : ils les tiendront pour vraies,/ Parce qu'elles ont l'apparence d'une vérité trompeuse./ Riens auxquels on accorde facilement foi, chansons. (...)"

42. P. Schede Melissus, 5-8: « (...) la Modestie au front pur/ accompagne [l'Amour], comme la Vénus vertueuse au cœur de neige./ L'Éther parfait l'engendra un jour/ Et l'envoya régner dans nos fibres enflammées. »

43. Lucrèce, De rerum natura, IV, 1058-1060.

44. P. Schede Melissus, 1-5: "S'il est dans la race humaine un nectar qui soit particulièrement enclin/ à captiver les esprits par ses charmes caressants,/ à instiller tant sa puissance que son Génie partout dans les moëlles,/ Et à les nourrir sans tarir, après avoir appâté les cœurs,/ C'est assurément l'Amour, Hagen. »

45. M. Ficin, Commentaire sur Le Banquet..., I, 3, 8-15.

46. P. Schede Melissus, 223-226: «Car parce qu'ils ont goûté une seule fois aux présents intacts/ de mes abeilles, nourries du suc de la rosée,/ Je ne sais de quelle propolis Amour fécond allaite ses poètes/ et ne leur permet pas d'oublier son rayon de miel. »

47. P. Schede Melissus, 71-72 : «Est-ce une vue de l'esprit ou bien le tendre Amour lui-même estil l'âme des Camènes,/ et le dénonciateur séduisant de leur vivant talent?»

\section{RÉSUMÉS}

Cet article est consacré aux pièces composées par le poète antique Ovide et le poète humaniste Paul Schede Melissus pour défendre la poésie érotique, dans un contexte de durcissement de la censure morale exercée à l'encontre la littérature. Nous avons choisi d'étudier successivement les deux stratégies qu'ils ont déployées, en tentant de mettre en évidence, pour le poète humaniste, les points sur lesquels il rejoint son prédécesseur antique, et ceux sur lesquels il apporte sa propre contribution. Dans les Tristes, Ovide a fondé son plaidoyer sur la revendication de la liberté qui devrait être laissée à l'auteur de fiction : c'est le seul endroit où Eros, de plus en plus encadré et réprimé dans la réalité par les lois Juliennes, peut conserver sa liberté de mouvement et sa puissance poétique. Pour Ovide, enfin, c'est l'aiguillon du désir qui éveille les facultés littéraires des auteurs et le plaisir des lecteurs. Dans les Schediasmata Poetica, le protestant Melissus s'est attaché à défendre les auteurs de vers érotiques qui l'ont précédé, dans 
l'Antiquité et à la Renaissance, en tentant de concilier leurs vers avec la doctrine chrétienne, interprétée par le néo-platonisme florentin. Le poète humaniste accorde un rôle prépondérant à l'élan amoureux dont il souligne la fécondité : c'est Eros qui assure une descendance aux couples dans le mariage et qui nourrit l'inspiration des poètes quand ils acceptent de sublimer leurs désirs érotiques dans la contemplation et la création artistiques.

Defence and Illustration of Love in poetry in Ovid's Tristia and in Paul Schede Melissus', neo-latin poet, Schediasmata Poetica

The present study is devoted to the poems composed by the Roman poet Ovid and the Humanist poet Paul Schede Melissus in order to defend erotic poetry during a period of higher moral censure against literature. We chose to analyse successively their strategies, to show which arguments the humanist poet borrows from the ancient one and which arguments he himself elaborates. In his plea, in the Tristia collection, Ovid claimed entire liberty for fiction : literature is the last place where Eros, increasingly supervised and suppressed in reality by Julian laws, can save its freedom of movement and poetic power. Lastly, according to Ovid, the spur of desire stimulate writers' literary abilities and readers' pleasure. In the Schediasmata Poetica, Melissus, a protestant poet, tried to defend erotic poets in Antiquity and Renaissance by conciliating their poetry and Christian doctrine, revisited by Florentine Neo-Platonism. The humanist poet emphasizes the importance and the fecundity of love's impulse: Eros gives descendants to couples and inspiration to poets when they agreed to transcend their erotic desires in artistic contemplation and creation.

\section{INDEX}

Keywords : Antiquity, censure, desire, erotic poetry, fiction, Humanism, plea

Mots-clés : Antiquité, censure, désir, fiction, Humanisme, plaidoyer, poésie érotique

\section{AUTEUR}

\section{KARINE DESCOINGS}

E.A. 4081- Université de Paris-Sorbonne 